\title{
Feature Selection for Ordinal Regression
}

\author{
Stefano Baccianella, Andrea Esuli and Fabrizio Sebastiani \\ Istituto di Scienza e Tecnologie dell'Informazione \\ Consiglio Nazionale delle Ricerche \\ Via Giuseppe Moruzzi, 1 - 56124 Pisa, Italy \\ firstname.lastname@isti.cnr.it
}

\begin{abstract}
Ordinal regression (also known as ordinal classification) is a supervised learning task that consists of automatically determining the implied rating of a data item on a fixed, discrete rating scale. This problem is receiving increasing attention from the sentiment analysis and opinion mining community, due to the importance of automatically rating increasing amounts of product review data in digital form. As in other supervised learning tasks such as (binary or multiclass) classification, feature selection is needed in order to improve efficiency and to avoid overfitting. However, while feature selection has been extensively studied for other classification tasks, is has not for ordinal regression. In this paper we present four novel feature selection metrics that we have specifically devised for ordinal regression, and test them on two datasets of product review data.
\end{abstract}

\section{Keywords}

Ordinal regression, ordinal classification, feature selection, product reviews

\section{INTRODUCTION}

Text management tasks such as ad hoc text retrieval, text clustering, and text classification, are usually tackled by representing the textual documents in vector form. The resulting vector spaces are always characterized by a high dimensionality (often in the range of the tens, sometimes hundreds of thousands dimensions), since words (or word stems) are normally used as features, and since many thousands of them occur in any reasonably-sized document space. This very high dimensionality is not very problematic in ad hoc retrieval, where the basic operation (computing the distance of two vectors in the vector space) can be performed quickly, thanks to the sparse nature of the two vectors. It is instead problematic in other tasks involving supervised or unsupervised learning, such as text classification or clustering.

For instance, in text classification many supervised learning devices, such as neural networks, do not scale well to

Permission to make digital or hard copies of all or part of this work for personal or classroom use is granted without fee provided that copies are not made or distributed for profit or commercial advantage and that copies bear this notice and the full citation on the first page. To copy otherwise, to republish, to post on servers or to redistribute to lists, requires prior specific permission and/or a fee.

SAC'10 March 22-26, 2010, Sierre, Switzerland.

Copyright 2010 ACM 978-1-60558-638-0/10/03 ...\$10.00. large numbers of features, and even those learning devices that do scale well have a computational cost at least linear in the dimensionality of the vector space. While this negatively impacts on efficiency, effectiveness suffers too, since if the ratio of the number of training examples to the number of features is low, overfitting occurs. For all these reasons, in text managements tasks involving learning the high dimensionality of the vector space may be problematic. Several techniques for reducing the dimensionality of a vector space for text learning tasks have been investigated, the main one being feature selection (FS). This latter consists in identifying a subset $S \subset T$ of the original feature set $T$ such that $|S| \ll|T|(\xi=|S| /|T|$ being called the reduction level $)$ and such that $S$ reaches the best compromise between (a) the effectiveness of the resulting classifiers and (b) the efficiency of the learning process and of the classifiers (which is, of course, inversely proportional to $|S|)$.

While feature selection mechanisms have been extensively investigated for text classification [4, 11], and to a lesser extent for text clustering, they have not for a related and important text learning task, namely, ordinal regression for text. Ordinal regression (OR - also known as ordinal classification) consists in estimating a target function $\Phi: X \rightarrow R$ which maps each object $x_{i} \in X$ into exactly one of an ordered sequence (that we here call rankset) $R=\left\langle r_{1} \prec\right.$ $\left.\ldots \prec r_{n}\right\rangle$ of ranks (aka "scores", or "labels", or "classes"), by means of a function $\hat{\Phi}$ called the classifier $^{1}$. This problem is somehow intermediate between single-label classification, in which $R$ is instead an unordered set, and metric regression, in which $R$ is instead a continuous, totally ordered set (typically: the set $\mathbb{R}$ of the reals). A key feature of ordinal regression is also that the "distances" between consecutive ranks may be different from each other.

OR is of key importance in the social sciences, since human judgments and evaluations tend to be expressed on ordinal (i.e., discrete) scales; an example of this is customer satisfaction data, where customers may evaluate a product or service on a scale consisting of the values Disastrous, Poor, Fair, Good, Excellent.

In this paper we address the problem of feature selection for OR. Here of course we are only interested in "filter" approaches to FS, i.e., approaches in which a mathematical function $f$ is applied to each feature in $T$ in order to compute its expected contribution to solving the classification task, after which only the $x=|S|$ top-scoring features are retained [7] ( $x$ may be a predetermined number or may,

\footnotetext{
${ }^{1}$ Consistently with most mathematical literature we use the caret symbol $\left(^{\wedge}\right)$ to indicate estimation.
} 
more typically, be expressed as a percentage of $|T|$ ). "Wrapper" approaches, in which entire subsets of $x$ features are evaluated as a whole through the actual learn-and-classify process, are instead not feasible in text-related applications, due to the high dimensionality of the feature space ${ }^{2}$. We here present four novel feature selection metrics that we have specifically devised for ordinal regression, and test them on two datasets of product review data.

The paper is organized as follows. In Section 2 we discuss related work, and in Section 3 we present our four FS algorithms for OR. Section 4 reports the results of experiments we have conducted on these methods, while Section 5 concludes.

\section{RELATED WORK}

To the best of our knowledge, there are only two papers [2, $9]$ that address feature selection for ordinal regression.

Mukras et al. [9] propose an algorithm, called probability redistribution procedure $(\mathrm{PRP})$, that takes as input the distribution of the feature across the ranks (as deriving from the distribution across the ranks of the training examples containing it) and modifies it, according to the notion that, when a feature $t_{k}$ occurs in (a document belonging to) a rank $r_{j}$, it is "taken to also occur", to a degree linearly decaying with the distance from $r_{j}$, in the ranks close to $r_{j}$. The modified distribution is then used in selecting features through a standard application, as in binary classification, of the information gain function.

Baccianella et al. [2] describe two methods called minimum variance (here noted $V a r$ ) and round robin on minimum variance $(R R(V a r))$. The basic idea underlying $V a r$ is that of measuring the variance of the distribution of a feature across the ranks, and retaining only the $t$ features that have the smallest variance. The intuition behind $V$ ar is that a feature is useful iff it is capable of discriminating a small, contiguous portion of the ordered scale from the rest, and that features with a small variance are those which tend to satisfy this property. $R R(V a r)$ is instead based on the idea (originally presented in [5] for binary text classification) that Var might select many features that discriminate well some of the ranks, while selecting few or no features that discriminate well the other ranks. In order to solve this, in $R R(\operatorname{Var})$ one provisionally "assigns" each feature $t_{k}$ to the rank closest to its average rank value, orders for each rank the features assigned to it, and then has the $n$ ranks take turns, according to a "round robin" (RR) policy, in picking features from the top-most elements of their rank-specific orderings.

\section{FEATURE SELECTION FOR ORDINAL CLASSIFICATION}

Let us fix some notation. As mentioned in Section 1, our task is to learn (from a training set $T r$ ) and evaluate (on a test set $T e$ ) a target function $\Phi: D \rightarrow R$ which classifies each document $d_{i} \in D$ into exactly one of an ordered sequence $R=\left\langle r_{1} \prec \ldots \prec r_{n}\right\rangle$ of ranks by means of a classifier $\hat{\Phi}$. Our feature selection methods will consist of scoring each feature $t_{k}$ by means of a scoring function Score that measures the predicted utility of $t_{k}$ for the classification process

\footnotetext{
${ }^{2}$ Interestingly, the literature on FS for metric regression seems to have mostly, if not only, investigated "wrapper" approaches [8].
}

(the higher the value of Score, the higher the predicted utility), and selecting the features based on their Score value.

\subsection{The Var*IDF method}

$\operatorname{Var} * I D F$ is a variant of the Var method described in [2].

Recall from Section 2 that $V a r$ is based on the intuition of retaining the features that minimize the variance of their distribution across the ranks. For instance, a feature $t_{k}$ that occurs only in (training documents belonging to) rank $r_{j}$ has, in the training set, variance $\operatorname{Var}\left(t_{k}\right)$ equal to zero; this feature seems obviously useful, since its presence in a test document $d_{i}$ can be taken as an indication that $d_{i}$ belongs to $r_{j}$. By the same token, a feature $t_{1}$ that occurs $90 \%$ of the times in $r_{j}$ and the remaining $10 \%$ in a rank contiguous to $r_{j}$ has lower variance than a feature $t_{2}$ that occurs $90 \%$ of the times in $r_{j}$ and the remaining $10 \%$ in a rank faraway from $r_{j}$. Feature $t_{1}$ is also more useful than $t_{2}$ since the presence of $t_{1}$ in a test document $d_{i}$ tends to indicate that $d_{i}$ belongs to $r_{j}$ or its vicinity, while $t_{2}$ gives a less clearcut indication. In sum, we are interested in retaining features with low variance and discarding ones with high variance.

However, we here note that a feature $t_{k}$ that occurs only once in the entire training set (e.g., in rank $r_{j}$ ) is trivially such that $\operatorname{Var}\left(t_{k}\right)=0$, but is not useful, since the fact that it occurs exactly in $r_{j}$ might be due to chance. The features that we are really interested in are those that have low variance and high frequency of occurrence (in the training set), since this high frequency of occurrence lends statistical robustness to the estimated value of their variance.

We formalize this by defining

$$
\operatorname{Score}\left(t_{k}\right)=-\operatorname{Var}\left(t_{k}\right) *\left(I D F\left(t_{k}\right)\right)^{a}
$$

where $I D F$ is the standard inverse document frequency, defined as $I D F\left(t_{k}\right)=\log \frac{|T r|}{\#_{T r}\left(t_{k}\right)}$ (where $\#_{T r}\left(t_{k}\right)$ denotes the number of training documents that contain feature $t_{k}$ ), and $a$ is a parameter (to be optimized on a validation set) that allows to fine-tune the relative contributions of variance and $I D F$ to the product. Note that, when $\operatorname{Var}\left(t_{k}\right)=0$, we still have $\operatorname{Score}\left(t_{k}\right)=0$, irrespectively of the value of $\operatorname{IDF}\left(t_{k}\right)$, which is undesirable. As a result, we smooth $\operatorname{Var}\left(t_{k}\right)$ by adding to it a small value $\epsilon=0.1$ prior to multiplying it by $I D F\left(t_{k}\right)$.

The $x$ features with the highest $S$ core value are retained while the others are discarded.

\subsection{The RR(Var*IDF) method}

Similarly to $\operatorname{Var}$, the $\operatorname{Var} * I D F$ method runs the risk of exclusively catering for a certain rank and disregarding the others. If all the retained features mostly occur in rank $r_{j}$ and its neighbouring ranks, the resulting feature set will exclusively contain features good at discriminating $r_{j}$ and its neighbouring ranks from the rest, while other ranks might not be adequately discriminated by any of the remaining features.

Similarly to the $R R($ Var $)$ method hinted at in Section 2, in order to pick the best $x$ features the $R R(\operatorname{Var} * I D F)$ method thus (i) provisionally "assigns" each feature $t_{k}$ to the rank closest to the mean of its distribution across the ranks; (ii) orders, for each rank $r_{j}$, the features provisionally assigned to $r_{j}$ in terms of their value of the Score function of Equation 1, with the highest-scoring ones at the top of the ordering; and (iii) enforces a "round robin" policy in which the $n$ ranks take turns, for $\frac{x}{n}$ rounds, in picking their 
favourite features from the top-most elements of their rankspecific orderings. In this way, for each rank $r_{j}$ the final set of selected features contains the $\frac{x}{n}$ features that are best at discriminating $r_{j}$ and its neighbouring ranks, which means that all the ranks in the rankset $R$ are adequately championed in the final feature set $S$.

\subsection{The RR(IGOR) method}

The round robin on information gain for ordinal regression $(R R(I G O R))$ method is based on the idea of adapting information gain (also known as mutual information), a function routinely employed in feature selection for binary classification (see e.g. [11]), to ordinal regression ${ }^{3}$.

In a binary classification task in which we need to separate class $c_{j}$ from its complement $\bar{c}_{j}$ we may perform feature selection by scoring each feature $t_{k}$ with the function

$$
\begin{aligned}
I G\left(t_{k}, c_{j}\right) & =H\left(c_{j}\right)-H\left(c_{j} \mid t_{k}\right)= \\
& =\sum_{c \in\left\{c_{j}, \bar{c}_{j}\right\}} \sum_{t \in\left\{t_{k}, \bar{t}_{k}\right\}} P(t, c) \log \frac{P(t, c)}{P(t) P(c)}
\end{aligned}
$$

where $H(\cdot)$ indicates entropy and $H(\cdot \mid \cdot)$ indicates conditional entropy. $I G\left(t_{k}, c_{j}\right)$ measures the reduction in the entropy of $c_{j}$ obtained as a result of $t_{k}$, i.e., measures the information that $t_{k}$ provides on $c_{j}$; for binary classification the $x$ features with the highest $I G\left(t_{k}, c_{j}\right)$ value are thus retained, while the others are discarded.

$R R(I G O R)$ is based on the idea of viewing ordinal regression on rankset $R=\left\langle r_{1} \prec \ldots \prec r_{n}\right\rangle$ as the simultaneous generation of $(n-1)$ binary classifiers $\ddot{\Phi}_{j}$, each of which is in charge of deciding whether a document belongs to one of the ranks $R_{j}=\left\{r_{1}, \ldots, r_{j}\right\}$ or to one of the ranks $\bar{R}_{j}=\left\{r_{j+1}, \ldots, r_{n}\right\}$, for $j=1, \ldots,(n-1)$. We call $R_{j}$ and $\bar{R}_{j}$ a rankset prefix and a rankset suffix, respectively. For each feature $t_{k}$ we thus compute $(n-1)$ different $I G\left(t_{k}, c_{j}\right)$ values, by taking $c_{j}=r_{1} \cup \ldots \cup r_{j}$ and $\bar{c}_{j}=r_{j+1} \cup \ldots \cup r_{n}$, for $j=1, \ldots,(n-1)$.

Similarly to the $R R(\operatorname{Var} * I D F)$ method of Section 3.2, in order to pick the best $x$ features we (i) order, for each of the $(n-1)$ binary classifiers $\ddot{\Phi}_{j}$ above, the features in terms of their value of the $I G\left(t_{k}, c_{j}\right)$ function, with the highestscoring ones at the top of the ordering; and (ii) enforce a round robin policy in which the $(n-1)$ classifiers $\ddot{\Phi}_{j}$ above take turns, for $\frac{x}{n-1}$ rounds, in picking their favourite features from the top-most elements of their classifier-specific orderings. In this way, for each classifier $\ddot{\Phi}_{j}$ the final set of selected features contains the $\frac{x}{n}$ features that are best at discriminating the rankset prefix $R_{j}$ from the rankset suffix $\bar{R}_{j}$, which means that all the classifiers $\ddot{\Phi}_{j}$ of rankset $R$ are adequately championed in the final feature set $S$.

Of course the intuition here is that, if test document $d_{i}$ belongs in rank $r_{j}$, classifiers $\ddot{\Phi}_{1}, \ldots, \ddot{\Phi}_{j-1}$ will be represented in $S$ by features that indicate $d_{i}$ to belong to their corresponding rankset suffixes $R_{1}, \ldots, R_{j-1}$, while classifiers $\ddot{\Phi}_{j}, \ldots, \ddot{\Phi}_{n-1}$ will be represented in $S$ by features that indicate $d_{i}$ to belong to their corresponding rankset prefixes $\bar{R}_{j}, \ldots, \bar{R}_{n-1}$.

\subsection{The RR(NC*IDF) method}

A problem with the methods we have proposed up to now, and with the ones mentioned in Section 2, is that none of them depends on (i.e., optimizes) the specific evaluation measure chosen for evaluating ordinal regression. The $R R(N C * I D F)$ method tries to address this shortcoming by including the chosen evaluation measure as a parameter, and directly optimizing it.

Assume that $E$ is the chosen evaluation measure, and that $E\left(\hat{\Phi}, d_{i}\right)$ represents the error that classifier $\hat{\Phi}$ makes in classifying $d_{i}$; e.g., if $\hat{\Phi}\left(d_{i}\right)=r_{1}, \Phi\left(d_{i}\right)=r_{2}$ and $E$ is absolute error (see Section 4.1.2), then $E\left(\hat{\Phi}, d_{i}\right)=\left|r_{1}-r_{2}\right|$. Let us define the negative correlation of a feature $t_{k}$ with a rank $r_{j}$ in the training set $\mathrm{Tr}$ as

$$
N C_{T r}\left(t_{k}, r_{j}\right)=\frac{\sum_{\left\{d_{i} \in T r \mid t_{k} \in d_{i}\right\}} E\left(\tilde{\Phi}_{j}, d_{i}\right)}{\left|\left\{d_{i} \in T r \mid t_{k} \in d_{i}\right\}\right|}
$$

where $\tilde{\Phi}_{j}$ is the "trivial" classifier that assigns all documents to the same rank $r_{j}$. In other words, $N C_{T r}\left(t_{k}, r_{j}\right)$ measures how bad an indicator of membership in rank $r_{j}$ feature $t_{k}$ is, where "bad" is defined in terms of the chosen error measure.

Let us define the rank $R\left(t_{k}\right)$ associated to a feature $t_{k}$ as

$$
R\left(t_{k}\right)=\arg \min _{r_{j} \in R} N C_{T r}\left(t_{k}, r_{j}\right)
$$

i.e., as the rank that is least negatively correlated with the feature. It would now be tempting to define $S$ core $\left(t_{k}\right)$ as $-N C_{T r}\left(t_{k}, R\left(t_{k}\right)\right)$, i.e., as the opposite of the negative correlation between $t_{k}$ and the rank $R\left(t_{k}\right)$ associated to it. This would seem reasonable since, given that $R\left(t_{k}\right)$ is the rank that $t_{k}$ best identifies, $-N C_{T r}\left(t_{k}, R\left(t_{k}\right)\right)$ measures how well it identifies it. While this is in principle reasonable, for the same reasons as outlined in Section 3.1 we need to compensate for the fact that $N C$ does not pay attention to frequency-of-occurrence considerations; this method might thus select features whose estimates are not statistically robust.

This leads us to defining the Score of a feature $t_{k}$ as

$$
\operatorname{Score}\left(t_{k}\right)=-N C_{T r}\left(t_{k}, R\left(t_{k}\right)\right) *\left(I D F\left(t_{k}\right)\right)^{a}
$$

where the $a$ parameter serves the same purpose as in Equation 1. Similarly to what happens in the $R R(\operatorname{Var} * I D F)$ and $R R(I G O R)$ methods, in order to select the best $x$ features we now apply a round robin policy in which each rank $r_{j}$ is allowed to pick, among the features such that $R\left(t_{k}\right)=r_{j}$, the $\frac{x}{n}$ features with the best Score.

Finally, note that for ranksets in which the distances between consecutive ranks are not always equal (this may be the case for a rankset consisting of ranks Poor, Good, Very Good, Excellent, since it might be argued that the distance between Poor and Good is higher than the distance between Very Good and Excellent), $R R(N C * I D F)$ is the only technique (among those discussed in this paper, including those from the literature) that allows bringing to bear these distances in the feature selection phase: one only needs to plug these distances into the $E$ measure used to define negative correlation.

\footnotetext{
${ }^{3}$ Any function routinely used for feature selection in binary classification, such as chi-square or odds ratio, could have been used here in place of information gain.
} 


\begin{tabular}{|c||c|c||r|c|c|c|c|}
\hline Dataset & $|T r|$ & $|T e|$ & 1 & 2 & 3 & 4 & 5 \\
\hline TripAdvisor-15763 & 10,508 & 5,255 & $3.9 \%$ & $7.2 \%$ & $9.4 \%$ & $34.5 \%$ & $45.0 \%$ \\
Amazon-83713 & 20,000 & 63,713 & $16.2 \%$ & $7.9 \%$ & $9.1 \%$ & $23.2 \%$ & $43.6 \%$ \\
\hline
\end{tabular}

Table 1: Main characteristics of the two datasets used in this paper; the last five columns indicate the fraction of documents that have a given number of "stars".
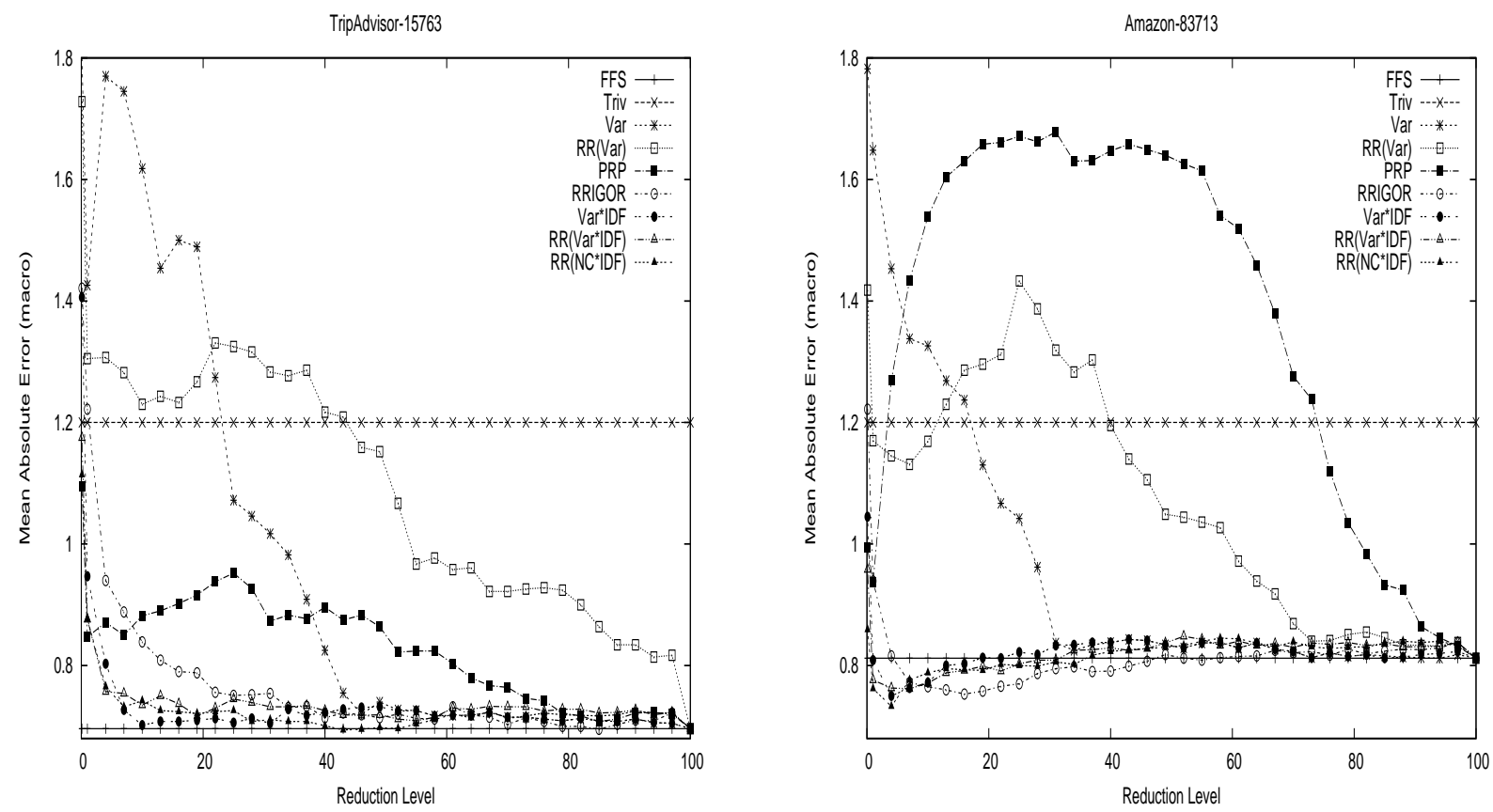

Figure 1: Results obtained with three baseline + four novel feature selection techniques on the TripAdvisor15763 dataset (left) and on the Amazon-83713 dataset (right). Results are evaluated with $M A E^{M}$; lower values are better. "FFS" refers to the full feature set (i.e., no feature selection), while "Triv" refers to uniform assignment to the trivial class.

\section{EXPERIMENTS}

\subsection{Experimental setting}

\subsubsection{The datasets}

We have tested the proposed measures on two different datasets, whose characteristics are summarized in Table 1.

The first is the TripAdvisor-15763 dataset built by Baccianella et al. [2], consisting of 15,763 hotel reviews from the TripAdvisor Web site ${ }^{4}$. We use the same split between training and test documents of [2], resulting in 10,508 documents used for training and 5,255 used for test; the training set contains 36,670 unique words. From the 10,508 training documents we have randomly picked 3,941 to be used as a validation set for parameter optimization.

\footnotetext{
${ }^{4}$ The dataset is available for download from http://patty. isti.cnr.it/ ^baccianella/reviewdata/
}

The second dataset is what we here call Amazon-83713, consisting of 83,713 product reviews from the Amazon Web site; Amazon-83713 is actually a small subset of the Amazon dataset (consisting of more than 5 million reviews) originally built by Jindal and Liu for spam review detection purposes [6], and contains all the reviews in the sections MP3, USB, GPS, Wireless 802.11, Digital Camera, and Mobile Phone. We have randomly picked 20,000 documents to be used for training, and use the remaining 63,713 documents for test; the training set contains 138,964 unique words. From the 20,000 training documents we have randomly selected 4,000 to be used as a validation set. To the best of our knowledge, Amazon-83713 is now the largest dataset ever used in the literature on rating product reviews.

Both datasets consist of reviews scored on a scale from 1 to 5 "stars"; both datasets are highly imbalanced (see Table 1), with positive and very positive reviews by far outnumbering negative and very negative reviews. 

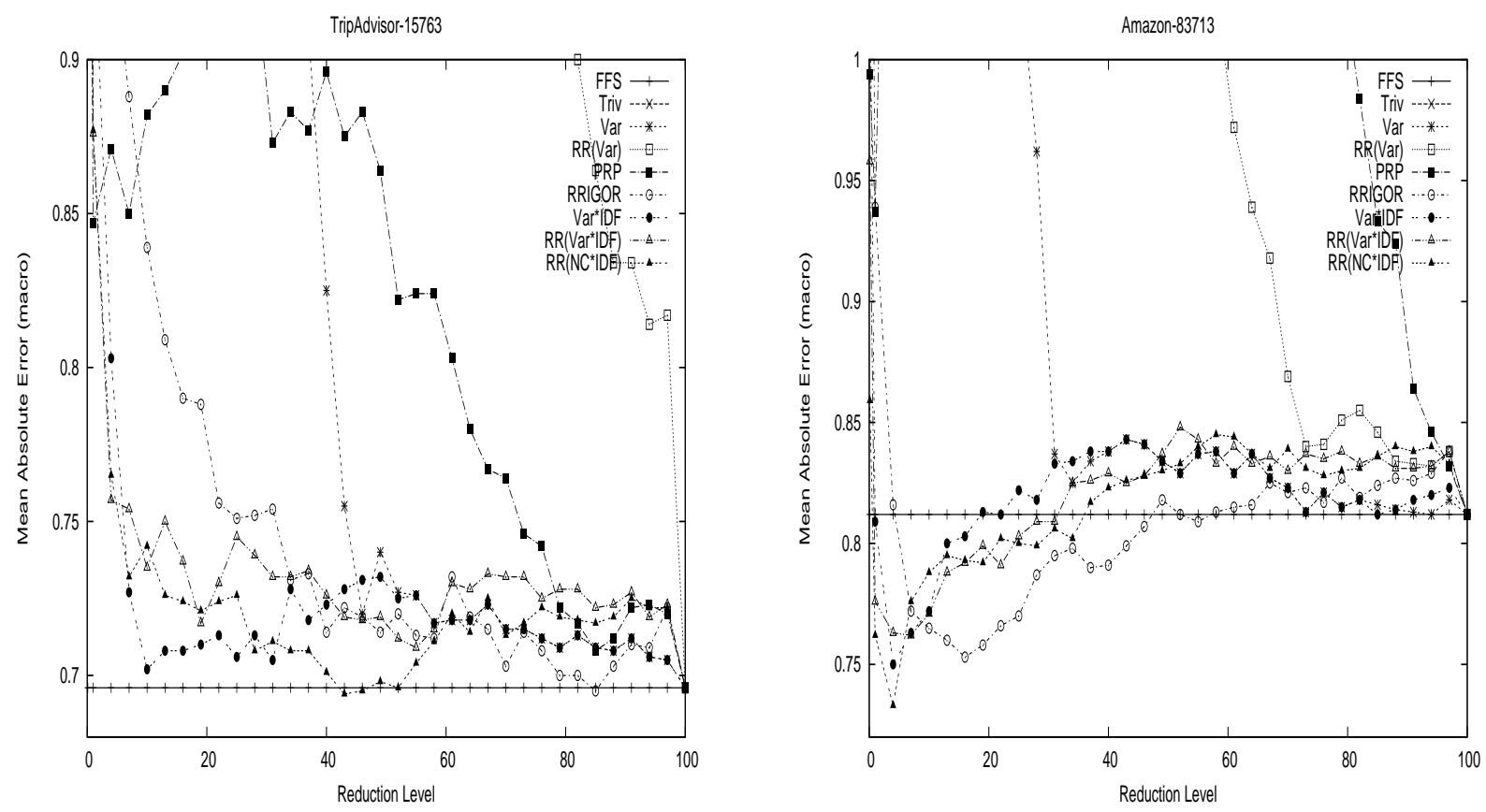

Figure 2: "Close-up" on the best results of Figure 1.

\subsubsection{Evaluation measures}

As our evaluation measure we use the macroaveraged mean absolute error $\left(M A E^{M}\right)$ measure proposed in [1], and defined as

$$
M A E^{M}(\hat{\Phi}, T e)=\frac{1}{n} \sum_{j=1}^{n} \frac{1}{\left|T e_{j}\right|} \sum_{d_{i} \in T e_{j}}\left|\hat{\Phi}\left(d_{i}\right)-\Phi\left(d_{i}\right)\right|
$$

where $T e_{j}$ denotes the set of test documents whose true rank is $r_{j}$ and the "M" superscript indicates "macroaveraging". As argued in [1], the advantage of $M A E^{M}$ over "standard" mean absolute error (defined as

$$
M A E^{\mu}(\hat{\Phi}, T e)=\frac{1}{|T e|} \sum_{d_{i} \in T e}\left|\hat{\Phi}\left(d_{i}\right)-\Phi\left(d_{i}\right)\right|
$$

where the " $\mu$ " superscript stands for "microaveraging") is that it is robust to rank imbalance (which is useful, given the above-mentioned imbalanced nature of our datasets) while coinciding with $M A E^{\mu}$ on perfectly balanced datasets (i.e., datasets with the same number of test documents for each rank).

\subsubsection{Baselines}

For all our experiments we have used three different baseline methods: the first is the PRP method of [9], while the second and third are the $\operatorname{Var}$ and $R R(\operatorname{Var})$ methods of [2] (see Section 2 for details).

We also draw comparisons with the "trivial baseline", i.e., with the method that consists in trivially assigning all test documents to the "trivial class", defined as follows. For a given (binary, ordinal, or other) classification problem a trivial classifier $\tilde{\Phi}_{j}$ may be defined as a classifier that assigns all documents to the same class $r_{j}$; accordingly, the trivial class $\tilde{r}$ may be defined as the class that minimizes the chosen error measure on the training set $T r$ across all trivial classifiers, i.e., $\tilde{r}=\arg \min _{r_{j} \in R} E\left(\tilde{\Phi}_{j}, T r\right)$, where $E$ is the chosen error measure. As argued in [1], the trivial class(es) for $M A E^{M}$ are always the middle classes, i.e. $r_{\left\lfloor\frac{n+1}{2}\right\rfloor}$ and $r_{\left\lceil\frac{n+1}{2}\right\rceil}$, which coincide with the 3 stars class in both the TripAdvisor-15763 and Amazon-83713 datasets (assignment to the trivial class yields $M A E^{M}=1.200$ for both datasets).

\subsubsection{Experimental protocol}

As a learning device for ordinal regression we have use $\epsilon$ support vector regression ( $\epsilon$-SVR) [10] as implemented in the freely available LibSvm library [3]. As a vectorial representation, after stop word removal (and no stemming) we use standard bag-of words with cosine-normalized $t$ fidf weighting. We have run all our experiments for all the 100 reduction levels $\xi \in\{0.001,0.01,0.02,0.03, \ldots, 0.99\}$.

For the $\operatorname{Var} * I D F, R R(\operatorname{Var} * I D F)$ and $R R(N C * I D F)$ methods we have (individually for each method) optimized the $a$ parameter on the validation set and then re-trained the optimized classifier on the full training set (i.e., including the validation set). During validation, all integer values in the range $[1,20]$ were tested (values smaller than 1 had already shown a dramatic deterioration in effectiveness, and were thus not investigated in detail), and the best value for a 
given method was retained. For all three methods this optimization was performed with $\xi=0.10$; further experiments on the validation set showed that in all cases the optimized parameter was optimal anyway for any $\xi \in[0.001,0.20]$.

For $R R(N C * I D F)$, the $E$ evaluation measure was taken to be $\left|\hat{\Phi}\left(d_{i}\right)-\Phi\left(d_{i}\right)\right|$ (i.e., absolute error), given that it is the document-level analogue of $M A E^{M}$.

\subsection{Results}

The results of our experiments are displayed in Figures 1 and 2 , in which the effectiveness of each feature selection policy is plotted as a function of the tested reduction level ${ }^{5}$. The two horizontal lines indicate the effectiveness obtained (a) by using the full feature set (i.e., no feature selection), or (b) by assigning all test documents to the trivial class.

In the discussion that follows we refer by default to the $M A E^{M}$ results on the Amazon-83713 dataset, since the test set of the Amazon-83713 dataset is more than 16 times larger than that of the TripAdvisor-15763 dataset, and we thus considers the results obtained on it somehow more reliable.

The first observation that comes natural by observing Figure 1 is that the three baselines are dramatically inferior to the four novel techniques proposed in this paper. $P R P$ is somehow comparable with our novel techniques for very aggressive reduction levels (e.g., $\xi=0.01$ ), but is drastically inferior to them for all other reduction levels, even underperforming the trivial baseline in the range $\xi \in[0.05,0.70]$ (it performs somehow better, bust still worse than our four proposed techniques, on TripAdvisor-15763). Var is, instead, comparable with our techniques for the less aggressive reduction levels (i.e., $\xi \in[0.4,1.0]$ ), but it yields very bad results for the more aggressive ones, even below the trivial baseline if $\xi \in[0.001,0.15]$; this is likely due to the fact that the top-scoring features for the $\operatorname{Var}$ method (i.e., the only ones that get selected when the reduction level is very aggressive) are features with very low frequency of occurrence (some of them maybe occurring in a single training document), while when the reduction level is less aggressive also "good" features (i.e., with low variance and high frequency of occurrence) are selected. $R R($ Var $)$ performs instead uniformly worse than the proposed techniques for all reduction levels and on both datasets. From now on we will thus largely ignore the three baseline techniques and focus on discussing our four novel techniques and their differences; in order to do this we will analyze Figure 2, which presents the same results of Figure 1 in close-up view, zooming-in on the best performing techniques.

A second observation (that comes especially evident by looking at Figure 1) is that our proposed techniques are fairly stable across $\xi \in[0.05,1.0]$, and deteriorate, sometimes rapidly, only for the very aggressive levels, i.e., for $\xi \in[0.001,0.05])$. This is in stark contrast with the instability of the baselines; e.g., as noted above, both PRP and Var perform reasonably well for some reduction levels but disastrously for others.

For $\xi \in[0.05,1.0]$ the accuracy is, for each of our four techniques, more or less comparable to the accuracy obtained with the full feature set (i.e., with no feature selection). The full feature set tends to be, although not by a wide margin, the best choice in the TripAdvisor-15763 dataset, while

\footnotetext{
${ }^{5}$ A spreadsheet with detailed figures on all the $7 \times 100 \mathrm{ex}-$ periments conducted can be downloaded at http://patty. isti.cnr.it/ baccianella/SAC10.xls.
}

the situation is less clearcut in the much larger Amazon83713 dataset, with the proposed techniques slightly underperforming the full feature set for $\xi \in[0.3,1.0]$, and outperforming it for $\xi \in[0.01,0.3]$. This latter is good news, since it indicates that one can reduce the feature set by an order of magnitude (with the ensuing benefits in terms of training-time and testing-time efficiency) and obtain an accuracy equal or even slightly superior (roughly a 10\% improvement, in the best cases) to that obtainable with the full feature set. Incidentally, this is clearly reminiscent of the results obtained by Yang and Pedersen, who, in their seminal paper on feature selection for text classification [11], showed that the best feature selection techniques could allow exactly that (i.e., an improvement in effectiveness of about $10 \%$ at $\xi=0.10$, which was the level at which the best performance was obtained).

It is not easy instead to decide which of the four techniques is best. On the Amazon-83713 dataset, $R R(I G O R)$ is clearly the best when $\xi \in[0.10,0.70]$, while when $\xi \in$ $[0.001,0.10]$ the best performer is $R R(N C * I D F)$. This latter even marginally outperforms the full feature set when the size of the feature set is reduced by two orders of magnitude, and only marginally underperforms it when the reduction is by three orders of magnitude; we think this is a striking performance. However, the situation is different on the TripAdvisor-15763 dataset, where $R R(N C * I D F)$ is the best performer for $\xi \in[0.3,0.6]$ but is beaten by $\operatorname{Var} * I D F$ when $\xi \in[0.1,0.3]$, a range in which (in stark contrast to Amazon-83713) $R R(I G O R)$ clearly underperforms the other three.

In order to get a clearer sense of the relative merits of these four techniques, in Table 2 we report the performance (for both datasets) of all the techniques discussed, averaged across the 50 reduction levels $\xi \in\{0.001,0.01,0.02, \ldots, 0.49$, $0.50\}$ (we consider that the reduction levels $\xi \in\{0.51, \ldots$, $0.99\}$ are scarcely interesting, since halving (or less) the dimensionality of one's feature space seems hardly worth the trouble of engaging in feature selection). The results reported show that $R R(I G O R)$ is the best performer (followed by $R R(N C * I D F)$ ), on both datasets, for our evaluation measure of choice $\left(M A E^{M}\right)$.

\section{CONCLUSIONS}

In this paper we have proposed four novel feature selection techniques for ordinal regression, and we have tested them against three baseline techniques from the literature on two datasets of product review data; one of these datasets is the largest dataset of product review data ever tested for ordinal regression purposes, and is being presented here for the first time.

The experiments, that we have carried out with thorough parameter optimization and for an extensive range of reduction levels, have clearly shown that all our four techniques are clearly superior to all three baselines, on both datasets. The experiments on the Amazon-83713 dataset (by far the larger of the two) seem to indicate that all techniques deliver a fairly stable performance across the range $[0.05,1.0]$ of reduction levels, and that performance tends to peak close to the 0.10 level; this indicates that it is viable to downsize the feature set by one order of magnitude while at the same time retaining, and sometimes even moderately improving upon, the effectiveness delivered by the full feature set.

In the future we plan to carry out further experiments in 


\begin{tabular}{|c|c|c|c|c|c|c|c|c|c|}
\hline & \multicolumn{5}{|c|}{ Baselines } & \multicolumn{4}{|c|}{ Our techniques } \\
\hline & 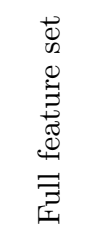 & 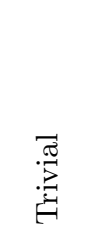 & $\delta$ & 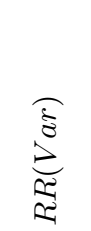 & $\frac{\hat{\alpha}}{\hat{\alpha}}$ & \begin{tabular}{l}
$\underset{1}{D}$ \\
\multirow{2}{*}{} \\
$\delta$ \\
$\delta$
\end{tabular} & 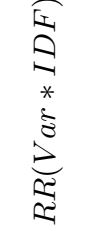 & $\begin{array}{l}\overparen{0} \\
0 \\
0 \\
- \\
02 \\
2\end{array}$ & 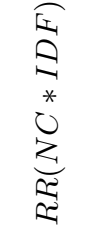 \\
\hline TripAdvisor-15763 & $\overline{0.696}$ & $\bar{~} 1.200$ & 1.200 & 1.255 & 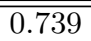 & $\overline{0.726}$ & 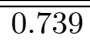 & 0.683 & $\overline{0.723}$ \\
\hline Amazon-83713 & 0.812 & 1.200 & 1.066 & 1.225 & 1.565 & 0.813 & 0.802 & 0.793 & 0.798 \\
\hline
\end{tabular}

Table 2: Performance of different feature selection functions as averaged across the reduction levels $\xi \in$ $\{0.001,0.01,0.02, \ldots, 0.49,0.50\}$. The best performer is indicated in boldface.

order to more clearly see which among the four proposed techniques is "the winner".

\section{Acknowledgements}

We thank Nitin Jindal and Bing Liu for kindly sharing with us their "Amazon" dataset, Xiubo Geng for clarifying the details of his experiments, and Salvatore Ruggieri for an important pointer to the literature.

\section{REFERENCES}

[1] S. Baccianella, A. Esuli, and F. Sebastiani. Evaluation measures for ordinal text classification. In Proceedings of the 9th IEEE International Conference on Intelligent Systems Design and Applications (ISDA'09), Pisa, IT, 2009.

[2] S. Baccianella, A. Esuli, and F. Sebastiani. Multi-facet rating of product reviews. In Proceedings of the 31st European Conference on Information Retrieval (ECIR'09), pages 461-472, Toulouse, FR, 2009.

[3] C.-C. Chang and C.-J. Lin. LIBSVM: a library for support vector machines, 2001. Software available at http://www.csie.ntu.edu.tw/ \{\} cjlin/libsvm.

[4] G. Forman. An extensive empirical study of feature selection metrics for text classification. Journal of Machine Learning Research, 3:1289-1305, 2003.

[5] G. Forman. A pitfall and solution in multi-class feature selection for text classification. In Proceedings of the 21st International Conference on Machine Learning (ICML'04), pages 38-45, Banff, CA, 2004.

[6] N. Jindal and B. Liu. Review spam detection. In Proceedings of the 16th International Conference on the World Wide Web (WWW'07), pages 1189-1190, Banff, CA, 2007.

[7] G. H. John, R. Kohavi, and K. Pfleger. Irrelevant features and the subset selection problem. In Proceedings of the 11th International Conference on Machine Learning (ICML'94), pages 121-129, New Brunswick, US, 1994.

[8] A. Miller. Subset selection in regression. Chapman and Hall, London, UK, second edition, 2002.

[9] R. Mukras, N. Wiratunga, R. Lothian, S. Chakraborti, and D. Harper. Information gain feature selection for ordinal text classification using probability re-distribution. In Proceedings of the IJCAI'O'7 Workshop on Text Mining and Link Analysis, Hyderabad, IN, 2007.

[10] B. Schölkopf, A. J. Smola, R. C. Williamson, and P. L. Bartlett. New support vector algorithms. Neural Computation, 12(5):1207-1245, 2000.

[11] Y. Yang and J. O. Pedersen. A comparative study on feature selection in text categorization. In Proceedings of the 14th International Conference on Machine Learning (ICML'97), pages 412-420, Nashville, US, 1997. 